\title{
A pilot observational study on application of nano size hyaluronic acids in relieving signs and symptoms on acute eczematous irritant facial skin eruptions
}

\author{
Chan KTM* \\ Specialist in Dermatology, Hong Kong SAR, Hong Kong, China
}

\begin{abstract}
Acute irritant eczematous facial skin eruption secondary to cosmetic procedures like laser therapy and inappropriate application of irritant skin care products are common clinical problems faced by dermatologists. Systemic and topical steroids, antibiotics, antihistamines are routinely prescribed but not without drawbacks like poor compliance, side effects like tachyphylaxis, steroid phobia and insomnolence. Doctor shopping is common and health seeking behaviour from non-evidence base practitioners is hazardous. The following is a report of using Nano-hyaluronic acid base serum to rapidly reduce acute irritant eczematous facial skin eruptions in patients in a clinic setting.
\end{abstract}

\section{Introduction}

Acute eczematous skin eruption over face is a common cause of dermatological clinic consultation. Many of these adverse skin reactions are secondary to acute irritative damage to the outer part of the epidermis; i.e. the stratum corneum (SC). The damaged protective layer can no longer prevent water from evaporation and an increased trans epidermal water loss (TEWL) resulted. Allergens and pathogens invasion may initiate an inflammatory cascade and directly sensitize the epidermal nerve free endings posited between the keratinocytes. Skin tautness, discomfort, burning sensation, pruritus and pain are common manifestations. Erythema and swelling complicated with crusts, oozes and scaling may aggregate the distressing symptoms with facial disfigurements made the already anxious sufferers more emotionally aroused and uncooperative.

An effective, safe, patient acceptable, rapid, non-invasive treatment should be examined in clinic setting. This would not only rapidly relieve the signs and symptoms of irritant facial eczematous eruption but may also gain patient rapport and confidence, improving doctorpatient relationship enhancing counselling and skin care education in subsequent consultations. This is important as a lot of these reactions may be secondary to individual behaviour of improper use of over the counter preparations of skin care and toiletries products containing irritative and sometimes toxic additives, preservatives, chemicals or microbial contaminants. While others are due to aesthetic procedures in beauty parlour like skin burnt secondary to lasers, radiofrequency and high intensity ultrasonic devices performed by untrained staffs. Theoretically, corticosteroids; either topical or systemic; should be considered first line therapeutics but in the wide spread misbelief of steroid phobia with its associated anxiety, moisturizers are good steroid sparing alternatives and adjuvants. But the large molecular size of some of these emollients may delay the recovery irritated skin processes due to its occlusive nature and poor permeability.

The following is a short pilot observational report on application of a Nano sized hyaluronic acid (N-HA) serum through cotton guaze soaked with sterilized water compress on five female patients who acutely presented with acute irritant eczematous facial skin eruption.

\section{Methodology}

\section{Patient selection}

In the month of August and September, 2018, altogether five patients with a diagnosis of acute eczematous facial skin eruption was recruited with consents. Their basic demography was shown in (Table 1). A detailed medical and drug history was documented. The history of sensitive skin was documented. The exclusion criteria are those who requested to be treated with topical or systemic steroids or other anti-inflammatories and those who are unwilling to be followed up and participated. Pretreatment photographs were taken with patient consents.

\section{The Nano Hyaluronic Acid serum (N-HA)}

The product used to apply over the affected face of the patient was called Hyalo-OligoR [1] with a molecular weight of 7000 by conversion to intrinsic viscosity. The average molecular size of the hyaluronic acid is between $15-25 \mathrm{~nm}$. The serum contained at least $1 \%$ of N-HA in the form of Hyalo-Oligo R plus other sizes of molecular weight of HA. The basic molecular structure is illustrated in (Figure 1).

\section{Methods}

Patients who agreed to receive the treatment have their face cleansed with a hypoallergenic face wash. A small amount of finger- tip

${ }^{\star}$ Correspondence to: Chan Kam Tim Michael, Specialist in Dermatology, Hong Kong SAR, Hong Kong, China, E-mail: pioneerskin@ymail.com

Key words: Acute irritant eczematous skin eruption, cosmesis, Nano-hyaluronic acid, rapid recovery, TWEL

Received: August 03, 2018; Accepted: August 07, 2018; Published: August 24 2018 

eruptions

Table 1. Basic demography and characteristics of the studied subjects

\begin{tabular}{|c|c|c|c|c|}
\hline Patient & Gender & Age & $\begin{array}{c}\text { Dermatological Clinical } \\
\text { Diagnosis }\end{array}$ & $\begin{array}{c}\text { History of Sensitive } \\
\text { Skin }\end{array}$ \\
\hline 1 & Female & 25 & Acute sun burnt & No \\
\hline 2 & Female & 27 & $\begin{array}{l}\text { Acute superficial burnt secondary } \\
\text { to picowave laser over left cheek }\end{array}$ & Yes \\
\hline 3 & Female & 40 & $\begin{array}{l}\text { Acute eczematous facial skin } \\
\text { eruption after applying self- } \\
\text { purchase cosmetics }\end{array}$ & Yes \\
\hline 4 & Female & 35 & $\begin{array}{l}\text { Acute pruritic left pre-auricular } \\
\text { skin eruption after hair dye }\end{array}$ & Yes \\
\hline 5 & Female & 43 & $\begin{array}{l}\text { Acute eczematous facial skin } \\
\text { eruption after laser therapy in } \\
\text { beauty parlour }\end{array}$ & No \\
\hline
\end{tabular}

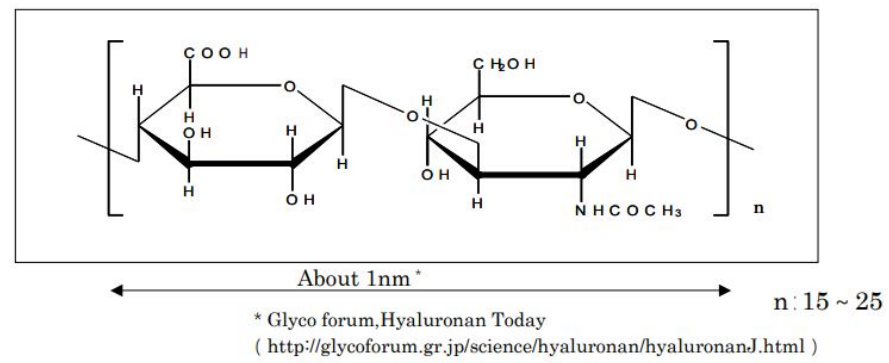

Figure 1. The basic molecular structure is illustrated

size of a serum $0.5 \mathrm{ml} \mathrm{N}$-HA was then applied to each side of the cheek, forehead, chin and the affected areas and gently messaged onto the skin surface by the clinic specialist nurse. After 10 minutes, cotton guaze pads soaked with sterile water was compressed to the treated areas to allow further deliverance of the N-HA to the deeper part of the epidermis. This is repeated for half an hour. The procedure was explained to the patient during the treatment. Post treatment photographs were taken with patient's consent. The patient was requested to be seen by the dermatologist after the procedure.

\section{Results}

The basic demography with the dermatological clinical diagnosis was shown in (Table 1). No reports of adverse reactions were reported. There was a reported reduction in eczematous sign and symptom objectively recorded by photographic documentation. The pre and post treatment photographs were illustrated in (Figure $2 \mathrm{a}, 2 \mathrm{~b}, 2 \mathrm{c}, 2 \mathrm{~d}$ and 2e). (Appendix) Patients also reported subjective improvements and acceptability of the treatments during the consultations.

\section{Discussion}

The epidermis is a complex structure with a natural non-viable stratum corneum interwoven into a basket-weaved histology pattern constantly turning over from bottom up through desquamation. Keratinocytes especially in the stratum granulosum contain lamellar bodies secreted an impermeable, lipid-containing membrane that maintain epidermal cohesion, control substrates permeability, antimicrobial, chemical, antioxidant defences. Interestingly, SC act also as an important percutaneous absorption organ through its aqueous pore formation for absorption of substances which is of nano dimension. [2] Lamellar bodies release lipids like ceramides, hydrolytic enzymes like proteases and proteins that are required for skin desquamation. $[3,4]$ Transportation of molecules via lamellar bodies is thought to prevent enzymes from interacting with their relevant substrates or inhibitors prior to secretion. [5] Irritant damage by environmental insults to the SC and lamellar bodies undoubtedly will result inflammation, pruritus and loss of skin natural morphophysiological functions.

The gap among cells of human skin surface is estimated as 40-50 $\mathrm{nm}$. [2]. Nano size particle in our case hyaluronic acid may be able to diffuse through these gaps and also the skin associated skin appendageal structures like the hair follicles and sweat gland apparatus. The damaged epidermis may also enhance a greater absorption coefficient in an already desiccated underlying actively injured epidermis. Besides, our method involved using sterile normal saline water as vehicle to
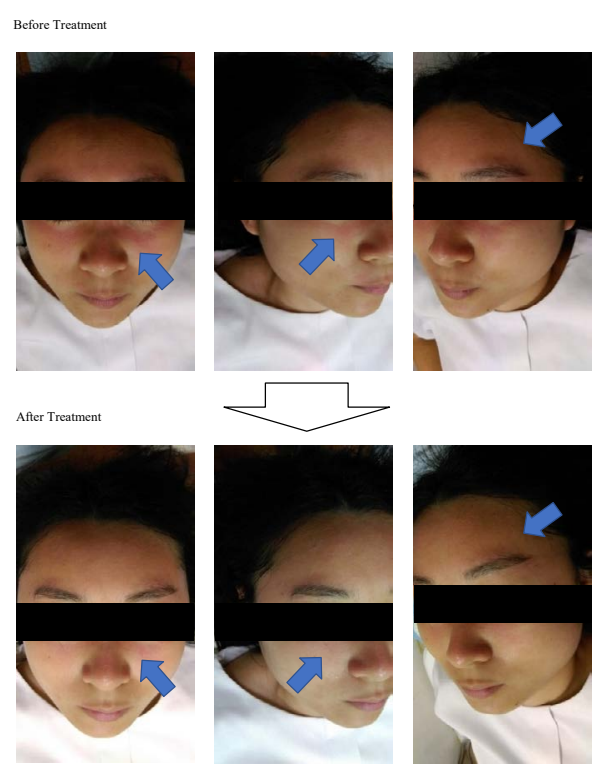

Figure 2a. Patient 1 (a patient suffered from sunburn) Patient case number: 020011, Treatment Date: 4-9-2018

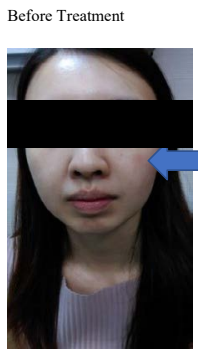

One Week Treatment
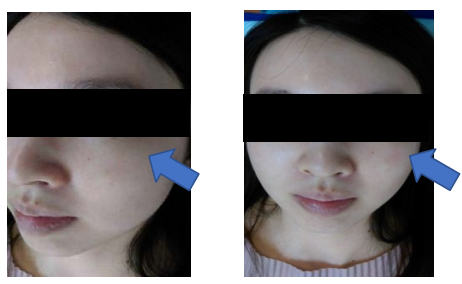

Two Week Treatment
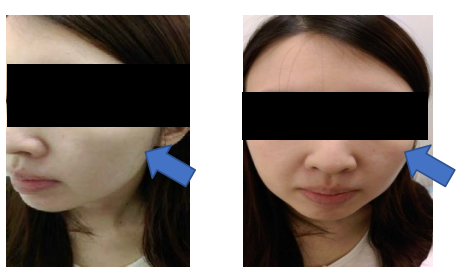

Figure 2b. Patient 2 (a patient suffered from superficial burnt after receiving pico laser in beauty palour) Patient case number: 017251, Treatment Date: 17-8-2018 

eruptions

assist transport of N-HA into the deeper part of the epidermis. The function of the N-HA is of three folds, firstly, it may act as a humectant or natural moisturizing factor (NMF) which bind surrounding water. Secondly, it blocks the SC defective barrier to prevent TWEL. Thirdly, the N-HA may act as a membrane that protect the already damaged SC. We postulate these as the possible underlying mechanisms on how the topical N-HA may reduce the signs and symptoms of acute eczematous facial eruption in our observed cases.

Hyaluronic acid has a good track record as a safe, non-toxic, biomimicking ground substances of many organs of our body including the skin. Indeed, aesthetic procedure involving injection of different particle size hyaluronic acid has been practiced, albeit not as safe as our topical administration route and the clinical purpose is different. N-HA, as we believed, should be one of the many candidates to consider to use in treating acute eczematous facial skin eruption with a rapid onset of efficacy, safety and patient acceptability. Furthermore, strictly speaking, $\mathrm{N}-\mathrm{HA}$ should not be regarded as a drug but prescription emollient devices (PED); its main component is part of the skin integral structure. Thus, it has the advantages of undergoing less vigorous and sometimes extensively prolonged testing and surveillance before ready to be used by the needed. In our preliminary, small number, observational clinical pilot study, N-HA when properly applied in clinically diagnosed irritant eczematous facial skin eruption is efficacious with no observable side effects and acceptable to the patients. Larger scale case control trials especially in comparing its effectiveness with topical corticosteroids
Before Treatment

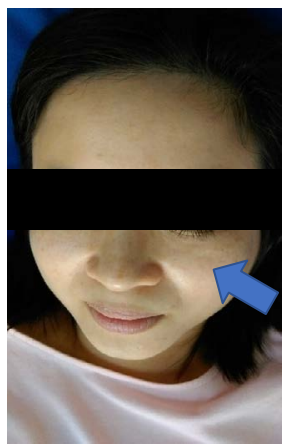

After Treatment

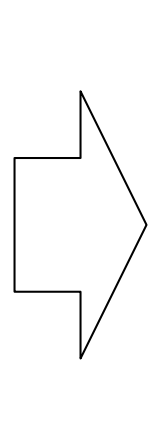

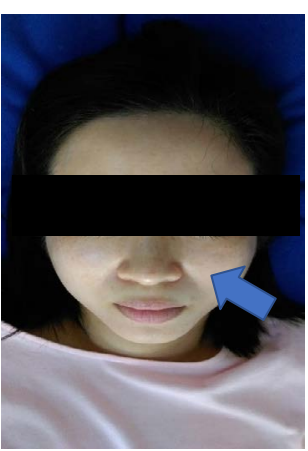

Figure 2c. Patient 3: A 40 year old female presented with acute erythematous pruritic left infraorbital skin eruption after applying a skin care products brought from the over the counter
Before Treatment

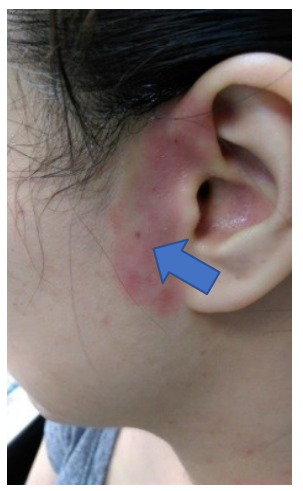

After Treatment

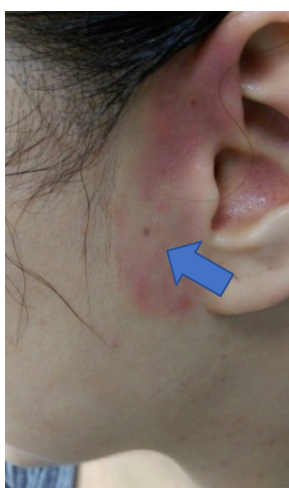

Figure 2d. Patient 4: A 35 year old female suffered from acute erythematous eczematous pruritic eruption over left anterior ear
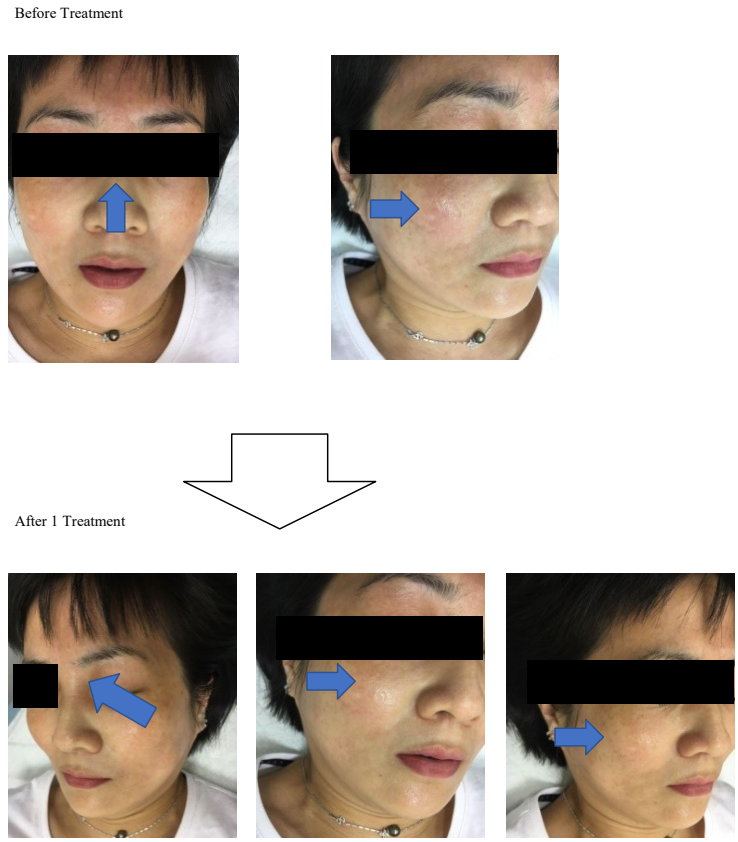

Figure 2e. Patient 5 (a patient received laser facial treatment for skin pigmentation developed acute eczematous facial skin eruption) Patient number: 19520, Treatment Date: 30-8-2018

in acute irritant eczema should be considered in future. Moreover, for conditions like sensitive skin syndrome in which the exact aetiology and pathogenesis is still obscure, N-HA may be a reasonable therapeutic treatment option to contemplate and study especially during the symptomatic stages on those with increase TWEL and thin SC.

In sum, we report a short observation case series of five female patients age 25 to 43 presented with acute eczematous irritant facial skin eruption which were treated effectively by N-HA serum using normal sterile saline water as vehicle. The main mechanism is to reduce TWEL and protect the damaged SC. More robust and detailed researches should be devoted to a greater number of patients suffered from this kind of iatrogenic acute facial skin eruption especially after incautious laser procedures and cosmeceutical products.

\section{References}

1. http://glycoforum.gr.jp/science/hyaluronan/hyaluronanJ.html

2. Al-Amoudi A, Dubochet D, Norlen L (2005) Nanostructure of the epidermal extracellular space as observed by cryo-electron microscopy of vitreous sections of human skin. J Invest Dermatol 124: 764-777. [Crossref]

3. Tortora and Derrickson, Gerard J. and Bryan H. Principles of anatomy and physiology. (2011) (13th ed.). Hoboken, N.J.: Wiley. p. 158. ISBN 978-0-470-64608-3.

4. Ishida-Yamamoto A, Kishibe Mari (2011) Involvement of corneodesmosome degradation and lamellar granule transportation in the desquamation process. Medical Molecular Morphology 44: 1-6. [Crossref]

5. Ishida-Yamamoto A, SimonM, Kishibe M, Miyauchi Y, Takahashi H, et al. (2004) Epidermal lamellar granules transport different cargoes as distinct aggregates. $J$ of Invest Dermatol 122: 1137-1144. [Crossref]

Copyright: (C2018 Chan KTM. This is an open-access article distributed under the terms of the Creative Commons Attribution License, which permits unrestricted use, distribution, and reproduction in any medium, provided the original author and source are credited. 